\title{
Glycopeptide Resistance in S. aureus
}

\author{
Hasan Cenk Mirza \\ Additional information is available at the end of the chapter
}

http://dx.doi.org/10.5772/65471

\begin{abstract}
The glycopeptides (particularly vancomycin) have been the recommended therapy for serious methicillin-resistant Staphylococcus aureus (MRSA) infections. The increased incidence of MRSA has led to the frequent use of vancomycin. Unfortunately, with the increased use of vancomycin, isolates of $S$. aureus have been discovered with reduced susceptibility to vancomycin. Several studies suggest that reduced vancomycin susceptibility is associated with vancomycin treatment failure. Various forms of glycopeptide resistance have appeared in MRSA strains, including high-level resistance, homogeneous and heterogeneous intermediate resistance. While vancomycin-resistant S. aureus (VRSA) strains are limited to a handful of reported cases and vancomycinintermediate $S$. aureus (VISA) strains remain rare; heterogeneous VISA (hVISA) strains are more common. This article summarizes the current knowledge regarding the history, definition, mechanisms, detection methods, epidemiology and clinical significance of 'glycopeptide resistance in S. aureus' and discusses therapeutic options for the treatment of hVISA/VISA infections.
\end{abstract}

Keywords: S. aureus, glycopeptide resistance, vancomycin, teicoplanin, hVISA, VISA

\section{Introduction}

Glycopeptide group antibiotics - notably vancomycin - have traditionally been the mainstay of therapy for infections caused by methicillin-resistant Staphylococcus aureus (MRSA) [1]. However, the increased incidence of MRSA infections has led to increased use of vancomycin and has resulted in the emergence of $S$. aureus with reduced susceptibility to vancomycin. Both the terms vancomycin-intermediate S. aureus (VISA) and glycopeptide-intermediate S. aureus (GISA) have been used in the literature [2]. Since many VISA isolates also have been intermediate to glycopeptide teicoplanin, the term GISA may be more accurate. However, the 
acronym VISA is more frequently used. Various studies have associated the presence of VISA and heterogeneous VISA (hVISA) with vancomycin treatment failure.

This article focuses on the history, definition, mechanisms, detection methods, epidemiology, and clinical significance of 'glycopeptide resistance in S. aureus' and the therapeutic options for the treatment of hVISA/VISA infections.

\section{History and definition of glycopeptide resistance}

The glycopeptide vancomycin was isolated from a Gram-positive filamentous actinomycete called Amycolatopsis orientalis and was approved for use by the U.S. Food and Drug Administration in 1958 [2, 3]. Vancomycin acts by inhibiting proper cell wall synthesis. It binds with high affinity to D-alanyl-D-alanine (D-Ala-D-Ala) terminal end of peptidoglycan precursors and prevents cross-linking of peptidoglycan by inhibiting the action of transglycosidase and transpeptidases. Vancomycin has been in clinical use for decades and there was no notable resistance to vancomycin reported in S. aureus until 1996. In 1996, a MRSA strain with vancomycin MIC of $8 \mu \mathrm{g} / \mathrm{ml}$ (Mu50, VISA) was isolated from the surgical wound infection from a 4month-old male infant who had undergone cardiac surgery [4]. After this case, two patients from USA and one patient from France with infections due to $S$. aureus with intermediate resistance to vancomycin were reported $[5,6]$. After the emergence of VISA; a new model of vancomycin resistance (hVISA) was defined by Hiramatsu et al. in 1997 [7]. The first hVISA strain Mu3 was isolated from the sputum of a 64-year-old patient with MRSA pneumonia who failed vancomycin therapy. hVISA strains are susceptible to vancomycin by the standard broth microdilution reference method (vancomycin MIC $\leq 2 \mu \mathrm{g} / \mathrm{ml}$ ) but contain subpopulations of cells (one in every $10^{5}-10^{6}$ ) for which the vancomycin MIC is in the intermediate range, currently defined as $4-8 \mu \mathrm{g} / \mathrm{ml}$ by the Clinical and Laboratory Standards Institute (CLSI) [8]. hVISA strains are more commonly found than VISA and different rates of hVISA are reported from different countries.

In 2002, the first S. aureus fully resistant to vancomycin [vancomycin-resistant S. aureus (VRSA), vancomycin MIC $\geq 16 \mu \mathrm{g} / \mathrm{ml}$ ] was reported in Michigan, United States (U.S). Fortunately, VRSA is very rare as only 14 cases of VRSA have been reported in U.S. so far [9].

\section{Phenotypic changes and mechanisms of resistance}

\section{1. hVISA and VISA}

Heterogeneous VISA appears to be the phase before the development of VISA. Vancomycin exhibits a selective pressure that leads to the growth of VISA subpopulations, eventually creating a uniform population of VISA [10].

One of the most common phenotypic changes observed in hVISA/VISA is the thickened cell wall with reduced peptidoglycan cross-linking (Figure 1) [2, 10, 11]. Reduced cross-linking of 
peptidoglycan leads to an increase in free D-Ala-D-Ala residues (binding sites for vancomycin). It is supposed that vancomycin binds to these free D-Ala-D-Ala residues in the outer layers of the thickened cell wall and is unable to reach its site of action at the cell membrane [12]. The trapped vancomycin molecules within the cell wall clog the peptidoglycan meshwork and form a physical barrier towards further incoming vancomycin molecules. Thus, collaboration of the clogging and cell wall thickening leads to glycopeptide resistance (Figure 2) [13, 14].

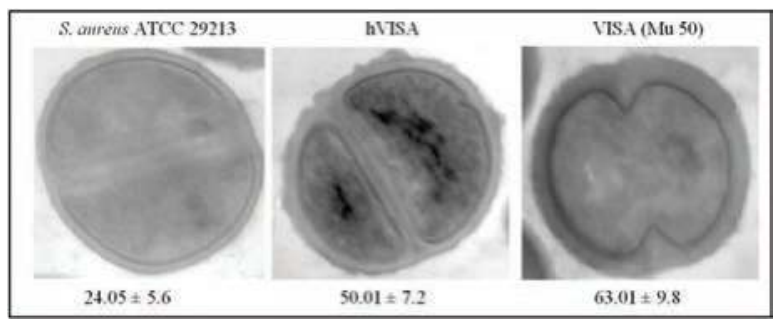

Figure 1. Comparison of the cell wall thickness of S. aureus ATCC 29213 [Vancomycin-susceptible S. aureus (VSSA)], hVISA and VISA strains by transmission electron microscopy. The cell wall thickness, in nanometers (mean \pm SD), is given under each image. Magnification: $\times 60,000$. (Adapted from Ref. [11] which was published under an open-access license agreement).

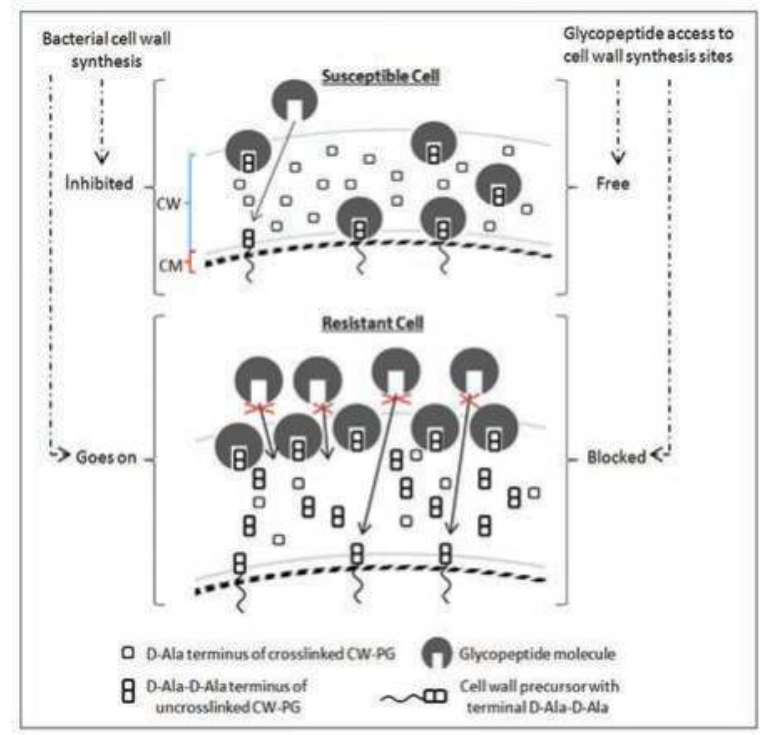

Figure 2. Model depicting the resistance mechanisms of hVISA/VISA. CW, cell wall; CM, cell membrane; PG, peptidoglycan. (Adapted with permission from Ref. [14]). 
In addition to thickened cell wall, hVISA/VISA strains exhibit other phenotypic changes including reduced autolytic activity, reduced hemolytic activity and slow growth in vitro $[10,15]$.

\subsubsection{Molecular mechanisms of resistance}

The molecular mechanisms of glycopeptide resistance in hVISA/VISA are still not clearly understood. To date, no specific genetic determinants of hVISA/VISA have been defined. However, some of the genes whose expression has been found to be altered in VISA strains include atl (autolysin), $\operatorname{mpr} F$ (phosphatidylglycerol lysyltransferase), sceD (transglycosylase), $\operatorname{sar} A, \operatorname{sig} B, t c a A$, and $d d h$ [16]. Furthermore, mutations associated with the intermediate resistance phenotype have been identified in the two-component regulatory systems [vraSR (vancomycin resistance-associated sensor/regulator), graSR (glycopeptide resistance-associated sensor/regulator), walKR], and rpoB (RNA polymerase) gene [15].

Cellular physiology of hVISA/VISA is believed to be altered due to the cumulative effects of mutations and/or modulation of regulatory systems [17]. As a result, altered cell wall structure and metabolism resulting from multiple genetic changes appears to be responsible for intermediate resistance to glycopeptides.

\subsection{VRSA}

The mechanism of vancomycin resistance in VRSA strains is different from that of hVISA/VISA strains. The vanA gene complex, which confers high-level resistance to glycopeptides in enterococci, was detected in VRSA isolates.

To date, nine types of glycopeptide resistance have been described in enterococci. Eight of these types (VanA, VanB, VanD, VanE, VanG, VanL, VanM, and VanN) correspond to acquired resistance, whereas VanC is an intrinsic characteristic of Enterococcus gallinarum and Enterococcus casseliflavus. Glycopeptide resistance in enterococci results from the production of modified peptidoglycan precursors ending in D-alanyl-D-lactate (D-Ala-D-Lac) (VanA, VanB, VanD, and VanM) or D-alanyl-D-serine (D-Ala-D-Ser) (VanC, VanE, VanG, VanL, and VanN) to which vancomycin binds with low affinity and the elimination of high-affinity precursors ending in D-Ala-D-Ala [18].

Resistance in VRSA isolates is caused by the horizontal transfer of transposon Tn1546 (carrying the vanA operon) from vancomycin-resistant Enterococcus faecalis [15, 19]. Vancomycin is known to act by binding to the terminal D-Ala-D-Ala of bacterial cell wall precursors. The vanA gene complex acquired by VRSA strains enables the bacteria to synthesize cell wall precursors terminating in D-Ala-D-Lac for which vancomycin has a greatly decreased affinity. In the presence of vancomycin, the novel cell wall precursors are synthesized, allowing continued peptidoglycan assembly [19, 20]. 


\section{Laboratory detection of hVISA, VISA and VRSA}

Laboratory detection of VISA and VRSA strains is easier than hVISA, as there are defined CLSI MIC criteria (MIC: $4-8 \mu \mathrm{g} / \mathrm{ml}$ for VISA and MIC $\geq 16 \mu \mathrm{g} / \mathrm{ml}$ for VRSA). These criteria have been defined using the reference broth microdilution (BMD) method. Results obtained by the use of other methods for determining the MIC should be confirmed with BMD [21]. Disk diffusion (Kirby-Bauer) is not an acceptable method for vancomycin susceptibility testing of S.aureus isolates.

Detection of hVISA is problematic for most clinical microbiology laboratories. The lack of a precise definition and standardized testing makes the detection of hVISA difficult [21]. hVISA strains appear susceptible to vancomycin (MIC $\leq 2 \mu \mathrm{g} / \mathrm{ml}$ ) with conventional testing but contain subpopulations ( 1 per $10^{5}-10^{6}$ organisms) that express reduced vancomycin susceptibility (MIC $\geq 4 \mu \mathrm{g} / \mathrm{ml}$ ). Standardized methods for susceptibility testing [broth microdilution (BMD), agar dilution and standard Etest] - which use an inoculum of only $5 \times 10^{4}$ colonyforming unit (CFU)/well (BMD) or $1 \times 10^{4} \mathrm{CFU} /$ spot (agar dilution) - fail to detect hVISA, in part due to the small inoculum, the relatively poor support of growth on Mueller-Hinton agar (MHA) plates, or a combination of both [2, 22].

The morphological features of hVISA/VISA isolates can be different from those of standard $S$. aureus cultures on agar plates. Careful observation may reveal smaller-sized colonies, "mixed" colony morphology (large and small colonies or colonies with different pigmentations in a pure culture) and reduced pigmentation. However, these changes may be subjective and are not diagnostic $[2,21]$.

Methods for "hVISA detection" use higher inoculum, prolonged incubation or more nutritious agar to promote the growth of subpopulations with reduced susceptibility to vancomycin. Population analysis profile-area under the curve (PAP-AUC) is considered the gold standard method for hVISA detection. However, this method is labor-intensive, time-consuming and not suitable for routine use in clinical microbiology laboratories [10, 22]. As a consequence, several screening methods have been developed for the detection of hVISA.

\subsection{Screening methods for hVISA}

\subsubsection{Screening plates}

A number of screening plates containing various concentrations of vancomycin or teicoplanin have been proposed for the detection of hVISA/VISA isolates [brain heart infusion agar (BHIA) with $3 \mu \mathrm{g} / \mathrm{ml}, 4 \mu \mathrm{g} / \mathrm{ml}$ or $6 \mu \mathrm{g} / \mathrm{ml}$ vancomycin, BHIA with $5 \mu \mathrm{g} / \mathrm{ml}$ teicoplanin, MHA with $5 \mu \mathrm{g} / \mathrm{ml}$ vancomycin or $5 \mu \mathrm{g} / \mathrm{ml}$ teicoplanin] [23-26]. Figure 3 shows the growth of a hVISA strain on BHIA with $4 \mu \mathrm{g} / \mathrm{ml}$ vancomycin. 


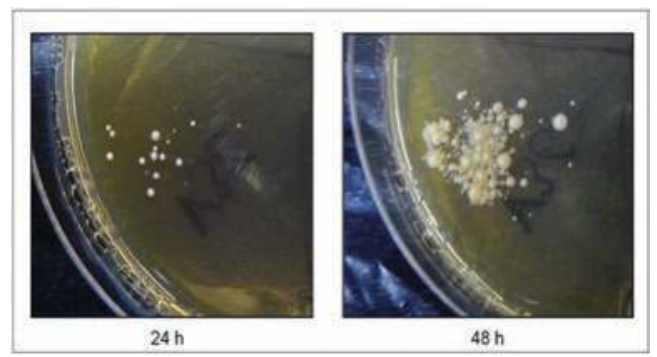

Figure 3. Photograph of a hVISA grown on BHIA with $4 \mu \mathrm{g} / \mathrm{ml}$ vancomycin for 24 and $48 \mathrm{~h}$.

Antibiogram Committee of the French Society for Microbiology recommends the use of MHA with $5 \mu \mathrm{g} / \mathrm{ml}$ teicoplanin (MHA5T). This screening plate has been tested by various studies using an inoculum of $10 \mu \mathrm{l}$ of a $2.0 \mathrm{McF}$ arland standard suspension for the detection of VISA/ hVISA isolates. Growth of one or more colonies is considered positive after $48 \mathrm{~h}$ of incubation. MHA5T has been shown to have sensitivity ranging from $65 \%$ to $79 \%$ and specificity ranging from 35 to $95 \%$ for the detection of hVISA [2].

In a study conducted by Satola et al., BHIA containing $4 \mu \mathrm{g} / \mathrm{ml}$ vancomycin and $16 \mathrm{~g} / \mathrm{l}$ pancreatic digest of casein has been shown to be $90 \%$ sensitive and $95 \%$ specific with a 0.5 McFarland inoculum and $100 \%$ sensitive and $68 \%$ specific with a 2.0 McFarland inoculum [22]. However, further studies are needed to determine the value of this screening plate.

\subsubsection{Etest macromethod}

Etest macromethod is performed utilizing a higher inoculum of organism (2.0 McFarland vs. 0.5 McFarland utilized in standard Etest) streaked onto BHIA. Vancomycin and teicoplanin Etest strips are applied to the dry agar surface and read after $48 \mathrm{~h}$ of incubation (compared to $24 \mathrm{~h}$ for standard Etest) at $35^{\circ} \mathrm{C}$ [10]. Zones must be read at complete inhibition, with care, to visualize hazy growth or microcolonies. Heteroresistance is defined as MICs for vancomycin and teicoplanin of $\geq 8 \mu \mathrm{g} / \mathrm{ml}$ or a teicoplanin MIC of $\geq 12 \mu \mathrm{g} / \mathrm{ml}$ alone. It should be noted that the result of the Etest macromethod is just a cutoff level and is not a true MIC, because this method differs from the standard MIC calculation [2, 21].

Figure 4 shows a hVISA strain (confirmed by PAP-AUC) with positive Etest macromethod result. The presence of microcolonies inside the inhibition zones reflects the heterogeneous resistant character of the strain (Figure 5). Subculture from a single microcolony (Figure 5B) done on blood agar reveals heterogeneous colony morphology (a common feature of hVISA/ VISA strains) (Figure 6).

Various studies have evaluated the performance of Etest macromethod, using PAP-AUC as the gold standard. Etest macromethod has been shown to have sensitivity ranging from 57 to $98.5 \%$ and specificity ranging from 55 to $96 \%$ for the detection of hVISA [22, 23, 25, 27-29]. The differences in sensitivity and specificity rates may be partially explained by the use of various 
inoculum sizes for Etest macromethod (50, 100 or $200 \mu \mathrm{l})$ in different studies [27, 28, 30]. Currently, the manufacturer recommends the use of an inoculum of $100 \mu \mathrm{l}$ [31].

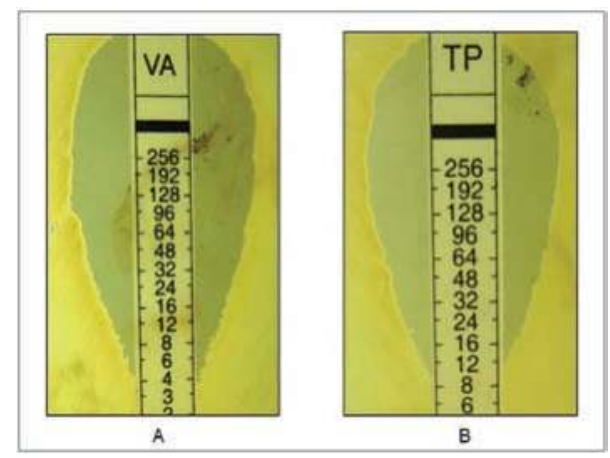

Figure 4. Positive Etest macromethod result for a hVISA strain isolated from blood culture A: Vancomycin Etest, B: Teicoplanin Etest.

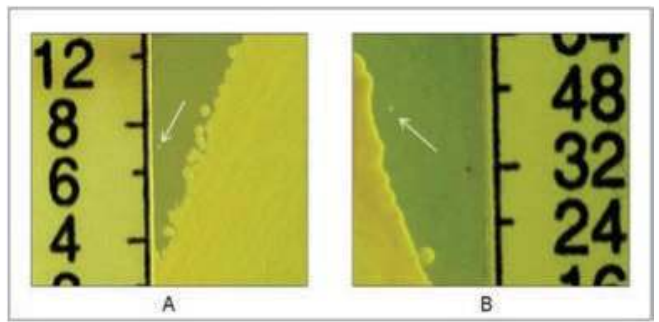

Figure 5. Magnified appearance of Figure 4(A and B). The arrows indicate the presence of microcolonies growing within the zones of inhibition. A: Vancomycin Etest, B: Teicoplanin Etest.

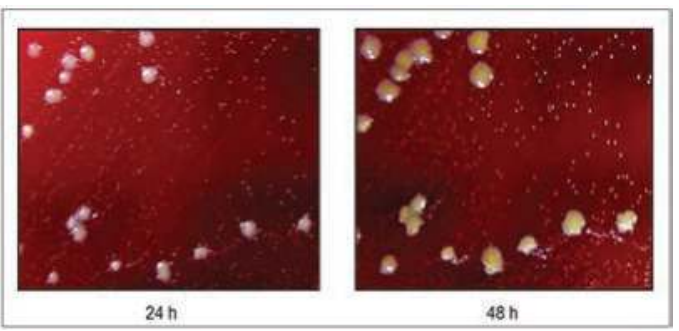

Figure 6. Subculture from a single microcolony (Figure 5B) on blood agar demonstrating different colony morphotypes. 


\subsubsection{Etest GRD}

Etest glycopeptide resistance detection (GRD) is a newer Etest method for the detection of hVISA. This method involves the use of a double-ended Etest strip that contains vancomycin, teicoplanin, and a nutritional supplement to enhance the growth of hVISA. A 0.5 McFarland standard inoculum, rather than the $2 \mathrm{McF}$ arland standard used for Etest macromethod, is used and inoculated onto MHA $+5 \%$ blood [2]. Etest GRD strip is applied to the agar surface and the zone of inhibition is read after $48 \mathrm{~h}$ of incubation at $35^{\circ} \mathrm{C}$. The strain is considered positive for hVISA if the Etest GRD result is $\geq 8 \mu \mathrm{g} / \mathrm{ml}$ for either vancomycin or teicoplanin [21, 22]. Figure 7 shows a hVISA strain with positive Etest GRD result.

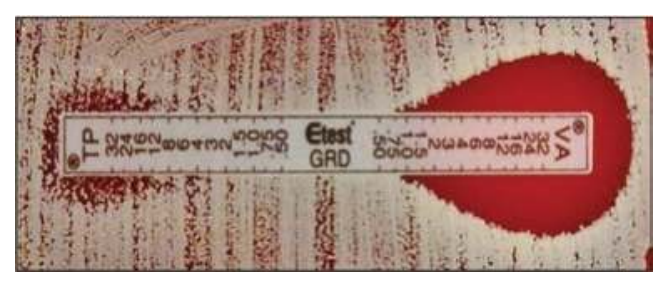

Figure 7. A hVISA strain with positive Etest GRD result. (Photograph courtesy of Dr M. Wootton).

Etest GRD has been reported to have sensitivity and specificity of $57-94 \%$ and $82-97 \%$, respectively $[22,28,29,32]$. Some of these differences may reflect the instability of hVISA phenotype. hVISA strains are known to be unstable, with the ability to revert to vancomycin-susceptible $S$. aureus (VSSA) under various conditions, including passage of the isolate on vancomycin-free media [29].

A possible barrier to large-scale usage of modified Etest methods is the potentially high cost to the clinical laboratory. This can be considered a disadvantage [10].

\subsection{Confirmatory methods for hVISA}

\subsubsection{PAP-AUC}

Population analysis profile-area under the curve (PAP-AUC) remains the gold standard method for detection of hVISA [10]. PAP-AUC method is performed as follows:

The isolate is incubated in tryptone soya broth (TSB) for $24 \mathrm{~h}$. An undiluted culture and dilutions of $1 / 10^{8}$ and $1 / 10^{5}$ are spiral plated onto BHIA plates containing $0.5,1,2,2.5,4$, and $8 \mu \mathrm{g}$ of vancomycin per $\mathrm{ml}$. After $48 \mathrm{~h}$ of incubation at $35^{\circ} \mathrm{C}$, the colonies are counted. $\log _{10}$ of the colony numbers $\left(\log _{10} \mathrm{CFU} / \mathrm{ml}\right)$ are plotted against the vancomycin concentrations. The "area under the curve (AUC)" of the isolate is calculated. The VSSA strain ATCC 29213 is used as negative control. Reference strains of hVISA (Mu3, ATCC 700698) and VISA (Mu50, ATCC 700699) are used as positive controls. A ratio is calculated by dividing the AUC of the test strain by the AUC of reference hVISA strain (Mu3). The ratios of $<0.90,0.90-1.30$, and $>1.30$ are interpreted as VSSA, hVISA, and VISA, respectively [28, 33]. Figure 8 shows an example 
of population analysis for S. aureus ATCC 29213 (VSSA), Mu3 (hVISA), Mu50 (VISA), a clinical hVISA isolate and a clinical VSSA isolate [11].

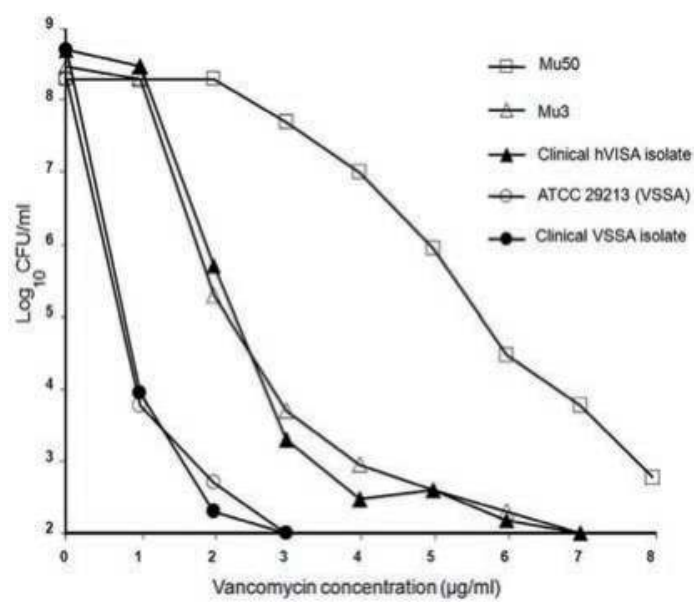

Figure 8. Example of population analysis profile curves for S. aureus ATCC 29213 (VSSA), Mu3 (hVISA), Mu50 (VISA), a clinical hVISA isolate and a clinical VSSA isolate. (Adapted from Ref. [11] which was published under an open-access licence agreement).

Unfortunately, PAP-AUC method is time-consuming, labor-intensive and costly which limits its use in routine clinical laboratories.

\section{Epidemiology}

While VRSA strains are limited to a handful of reported cases (14 total cases of VRSA in the U.S.) and VISA strains remain rare; hVISA strains are more common $[9,12,34]$. The true prevalence of hVISA is unknown, and estimates vary widely due to nonstandardized detection methods or absence of routine hVISA screening, variation in interpretation, clinical setting, geographical region, and differing patient populations [35]. Global hVISA rates among MRSA isolates have been reported to range from 0 to $73.7 \%$ [36].

In many studies, only the isolates suspected of being hVISA by screening methods (screening plates, Etest macromethod, Etest GRD) have been subjected to PAP-AUC. Some hVISA isolates may have been missed by screening methods in these studies. This may lead to an underestimation of the true prevalence $[2,27]$.

Although reported predominantly for MRSA; hVISA/VISA can be detected among methicillin-susceptible S. aureus (MSSA) strains [2,37]. However, routine testing of MSSA isolates for the presence of hVISA/VISA for clinical purposes is not necessary and not recommended [10]. 
High-level resistance to vancomycin in S. aureus is very rare and all VRSA strains reported to date have been MRSA [9].

The proportion of $S$. aureus isolates demonstrating heteroresistance increases with increasing vancomycin MICs within the susceptible range, but heteroresistance has been reported in strains with MICs as low as $0.5 \mu \mathrm{g} / \mathrm{ml}[21,38]$.

The main risk factors for hVISA and VISA infection appear to be prior MRSA colonization or infection and exposure to vancomycin. Most of hVISA/VISA infections occur in patients with serious underlying diseases such as malignancy, renal failure and diabetes, or in patients who have undergone major surgery $[2,12]$. Nosocomial spread and rare outbreaks caused by VISA or hVISA have also been reported [34].

\section{Clinical significance of hVISA/VISA and VRSA}

The clinical significance of hVISA/VISA is difficult to determine due to differences in definitions and laboratory detection as well as the lack of well-controlled prospective studies [2, 21]. Commonly reported associations with hVISA/VISA infections include vancomycin treatment failure and high-inoculum infections such as bacteremia, endocarditis, deep abscesses, osteomyelitis, and prosthetic device infections [2, 21, 35, 36]. Some authors consider that hVISA/VISA could be responsible for treatment failure, whereas others have suggested that it has arisen as a consequence of treatment failure and prolonged vancomycin exposures [39]. It is difficult to determine, especially if it is not clear when the VISA or hVISA isolate was detected in the course of infection [21].

Interestingly, attenuated virulence of $S$. aureus with reduced susceptibility to vancomycin has been reported in some animal infection models [40,41]. Pooled data from a meta-analysis showed similar mortality rates for hVISA and VSSA infections [36]. However, two recent studies have found a link between hVISA and higher mortality rate. Claeys et al. reported that patients with hVISA pneumonia experienced significantly higher inpatient mortality than those with VSSA pneumonia [42]. Hu et al. demonstrated that patients with hVISA bacteremia had a significantly higher in-hospital mortality than those with VSSA bacteremia [43].

The clinical significance of VRSA is unclear as only a few cases have been reported to date.

Persistent signs of infection and positive cultures for MRSA despite the administration of glycopeptide therapy, or relapse of infection after glycopeptide therapy can suggest an infection with hVISA or VISA [12]. Well-designed, large-scale prospective studies are needed to evaluate the clinical significance of these strains.

\section{Therapeutic options}

\subsection{Role of surgery}

Many patients with infections due to hVISA/VISA have high-inoculum infections (endocarditis, deep abscesses, osteomyelitis/septic arthritis and prosthetic device infections) $[2,12,36]$. 
Surgery is a useful adjunct to antimicrobial therapy for these patients. Main types of surgery include the drainage of abscesses or infected joints and removal of the infected prostheses [12].

\subsection{Antimicrobial therapy}

The emergence of hVISA/VISA clinical isolates has prompted the search for new antibiotics. While there are no guidelines regarding alternative antimicrobial therapy, there are a number of antimicrobial agents that have potential to be used in treatment of hVISA/VISA infections.

\subsubsection{Daptomycin}

Daptomycin is a lipopeptide antibiotic with activity against Gram-positive bacteria. In a study conducted by Wootton et al., despite slightly raised MICs seen for strains with reduced susceptibility to vancomycin; daptomycin showed greater bactericidal activity than vancomycin for hVISA and VISA [44]. This indicates that, while there is a potential for crossresistance between daptomycin and vancomycin, susceptibility to daptomycin is minimally affected by the presence of hVISA or VISA [10]. In several studies, daptomycin has been shown to have a good antimicrobial activity against the majority of hVISA isolates [27, 44, 45]. The highest rate of daptomycin nonsusceptibility was reported in a study evaluating 47 isolates of hVISA/VISA. In this study, the percentage of daptomycin nonsusceptible isolates was $15 \%$ for hVISA and 38\% for VISA [35, 46]. Additionally, daptomycin has been shown to have in vitro activity against VRSA isolates. In a study conducted by Sievert et al., one daptomycin nonsusceptible isolate was observed among 7 VRSA [47].

\subsubsection{Linezolid}

Linezolid is a synthetic antibacterial agent of the oxazolidinone class. Although resistance to linezolid has been reported in S. aureus isolates, rates of resistance remain very low. Linezolid was found to be useful for the treatment of hVISA/VISA infections [2, 48]. Also, data from a study demonstrated potent in vitro activity for linezolid against VRSA strains [47]. However, treatment with linezolid may be limited by toxicity. High rates of adverse reactions have been found for complex patients (seriously ill patients with multiple comorbidities) who received prolonged linezolid therapy [49]. Therefore, prolonged therapy should be used with caution in this patient group.

\subsubsection{Tigecycline}

Tigecycline is a glycylcycline antibiotic for intravenous infusion. In a study conducted by Sun et al., $26 \mathrm{hVISA}$ isolates and 1 VISA isolate were tested for tigecycline susceptibility. All isolates were found to be susceptible to tigecycline [50]. In another study, in vitro activity of tigecycline was evaluated against 33 VISA and 13 VRSA isolates. Tigecycline susceptibility rates were 97 and $92 \%$ for VISA and VRSA, respectively [51]. In vitro data have shown that tigecycline is active against hVISA/VISA as well as VRSA. Clinical studies are needed to determine the role of tigecycline in infections caused by $S$. aureus with reduced susceptibility to vancomycin. 


\title{
7.2.4. New cephalosporins
}

New cephalosporins like ceftaroline and ceftobiprole have been shown to be active against hVISA and VISA in vitro and in animal studies. In rabbit models of endocarditis, these agents were superior to vancomycin against hVISA and VISA [2]. While results of in vitro and in vivo testing of these cephalosporins have been positive against hVISA/VISA, their clinical utility for infections caused by hVISA or VISA remains unknown [10].

\subsubsection{Other antimicrobial agents}

Other potentially active antimicrobials include lipoglycopeptides (dalbavancin, oritavancin, telavancin), quinupristin-dalfopristin, rifampin and fusidic acid. However, resistance develops rapidly with monotherapy with rifampin or fusidic acid. Therefore, these agents should be used in combination with another antistaphylococcal agent. The combination of rifampin and fusidic acid is an effective option [2]. Studies also suggest the potential for synergistic activity between vancomycin and various antimicrobials including beta-lactams and gentamicin against $S$. aureus with reduced vancomycin susceptibility [10].

\section{Author details}

\author{
Hasan Cenk Mirza
}

Address all correspondence to: h_cenkmirza@yahoo.com.tr

Department of Microbiology, Merzifon State Hospital, Amasya, Turkey

\section{References}

[1] Appelbaum PC. The emergence of vancomycin-intermediate and vancomycin-resistant Staphylococcus aureus. Clin Microbiol Infect. 2006; 12 (Suppl 1):16-23. DOI: 10.1111/j. 1469-0691.2006.01344.x

[2] Howden BP, JK Davies, PD Johnson et al. Reduced vancomycin susceptibility in Staphylococcus aureus, including vancomycin-intermediate and heterogeneous vancomycin-intermediate strains: resistance mechanisms, laboratory detection, and clinical implications. Clin Microbiol Rev. 2010; 23:99-139. DOI: 10.1128/CMR.00042-09

[3] Xu L, Huang H, Wei W et al. Complete genome sequence and comparative genomic analyses of the vancomycin-producing Amycolatopsis orientalis. BMC Genomics. 2014; 15:363. DOI: $10.1186 / 1471-2164-15-363$ 
[4] Hiramatsu K, Hanaki H, Ino T et al. Methicillin resistant Staphylococcus aureus clinical strain with reduced vancomycin susceptibility. J Antimicrob Chemother. 1997; 40:135136. DOI: $10.1093 / \mathrm{jac} / 40.1 .135$

[5] Smith TL, Pearson ML, Wilcox KR et al. Emergence of vancomycin resistance in Staphylococcus aureus. N Engl J Med. 1999; 340:493-501. DOI: 10.1056/NEJM19990218 3400701

[6] Ploy MC, Grelaud C, Martin C. First clinical isolate of vancomycin-intermediate Staphylococcus aureus in a French hospital. Lancet. 1998; 351:1212. DOI: 10.1016/S01406736(05)79166-2

[7] Hiramatsu K, Aritaka N, Hanaki H et al. Dissemination in Japanese hospitals of strains of Staphylococcus aureus heterogeneously resistant to vancomycin. Lancet. 1997; 350:1670-1673. DOI: 10.1016/S0140-6736(97)07324-8

[8] Tenover FC. The quest to identify heterogeneously resistant vancomycin-intermediate Staphylococcus aureus strains. Int J Antimicrob Agents. 2010; 36:303-306. DOI: 10.1016/ j.ijantimicag.2010.06.005

[9] Walters MS, Eggers P, Albrecht V et al. Vancomycin-resistant Staphylococcus aureus Delaware, 2015. MMWR Morb Mortal Wkly Rep. 2015; 64:1056. DOI: 10.15585/ mmwr.mm6437a6.

[10] Rong SL, Leonard SN. Heterogeneous vancomycin resistance in Staphylococcus aureus: a review of epidemiology, diagnosis, and clinical significance. Ann Pharmacother. 2010; 44:844-850. DOI: 10.1345/aph.1M526

[11] Sola C, Lamberghini RO, Ciarlantini M et al. Heterogeneous vancomycin-intermediate susceptibility in a community-associated methicillin-resistant Staphylococcus aureus epidemic clone, in a case of Infective Endocarditis in Argentina. Ann Clin Microbiol Antimicrob. 2011; 10:15. DOI: 10.1186/1476-0711-10-15

[12] Howden BP. Recognition and management of infections caused by vancomycinintermediate Staphylococcus aureus (VISA) and heterogenous VISA (hVISA). Intern Med J. 2005; 35:S136-S140. DOI: 10.1111/j.1444-0903.2005.00986.x

[13] Cui L, Iwamoto A, Lian JQ et al. Novel mechanism of antibiotic resistance originating in vancomycin-intermediate Staphylococcus aureus. Antimicrob Agents Chemother. 2006; 50:428-438. DOI: 10.1128/AAC.50.2.428-438.2006

[14] Sieradzki K, Pinho MG, Tomasz A. Inactivated pbp4 in highly glycopeptide-resistant laboratory mutants of Staphylococcus aureus. J Biol Chem. 1999; 274:18942-18946. DOI: 10.1074/jbc.274.27.18942

[15] Deresinski S. The multiple paths to heteroresistance and intermediate resistance to vancomycin in Staphylococcus aureus. J Infect Dis. 2013; 208:7-9. DOI: 10.1093/infdis/ jit136 
[16] Samanta D, Elasri MO. The msaABCR operon regulates resistance in vancomycinintermediate Staphylococcus aureus strains. Antimicrob Agents Chemother. 2014; 58:6685-6695. DOI: 10.1128/AAC.03280-14

[17] Sakoulas G, Moellering RC Jr. Increasing antibiotic resistance among methicillinresistant Staphylococcus aureus strains. Clin Infect Dis. 2008; 46 (Suppl 5):S360-S367. DOI: $10.1086 / 533592$

[18] Garrido AM, Gálvez A, Pulido RP. Antimicrobial resistance in Enterococci. J Infect Dis Ther. 2014; 2:150. DOI: 10.4172/2332-0877.1000150

[19] Gardete S, Tomasz A. Mechanisms of vancomycin resistance in Staphylococcus aureus. J Clin Invest. 2014; 124:2836-2840. DOI: 10.1172/JCI68834

[20] Lowy FD. Antimicrobial resistance: the example of Staphylococcus aureus. J Clin Invest. 2003; 111:1265-1273. DOI: 10.1172/JCI200318535

[21] Holmes NE, Johnson PD, Howden BP. Relationship between vancomycin-resistant Staphylococcus aureus, vancomycin-intermediate S. aureus, high vancomycin MIC, and outcome in serious S. aureus infections. J Clin Microbiol. 2012; 50:2548-2552. DOI: 10.1128/JCM.00775-12

[22] Satola SW, Farley MM, Anderson KF et al. Comparison of detection methods for heteroresistant vancomycin-intermediate Staphylococcus aureus, with the population analysis profile method as the reference method. J Clin Microbiol. 2011; 49:177-183. DOI: $10.1128 / J C M .01128-10$

[23] Wootton M, MacGowan AP, Walsh TR et al. A multicenter study evaluating the current strategies for isolating Staphylococcus aureus strains with reduced susceptibility to glycopeptides. J Clin Microbiol. 2007; 45:329-332. DOI: 10.1128/JCM.01508-06

[24] Riederer K, Shemes S, Chase P et al. Detection of intermediately vancomycin-susceptible and heterogeneous Staphylococcus aureus isolates: comparison of Etest and Agar screening methods. J Clin Microbiol. 2011; 49:2147-2150. DOI: 10.1128/JCM.01435-10

[25] Voss A, Mouton JW, van Elzakker EP et al. A multi-center blinded study on the efficiency of phenotypic screening methods to detect glycopeptide intermediately susceptible Staphylococcus aureus (GISA) and heterogeneous GISA (h-GISA). Ann Clin Microbiol Antimicrob. 2007; 6:9. DOI: 10.1186/1476-0711-6-9

[26] Fitzgibbon MM, Rossney AS, O'Connell B. Investigation of reduced susceptibility to glycopeptides among methicillin-resistant Staphylococcus aureus isolates from patients in Ireland and evaluation of agar screening methods for detection of heterogeneously glycopeptide-intermediate S. aureus. J Clin Microbiol. 2007; 45:3263-3269. DOI: 10.1128/ JCM.00836-07

[27] Mirza HC, Sancak B, Gür D. The Prevalence of vancomycin-intermediate Staphylococcus aureus and heterogeneous VISA among methicillin-resistant strains isolated from 
pediatric population in a Turkish University Hospital. Microb Drug Resist. 2015; 21:537544. DOI: $10.1089 / \mathrm{mdr} .2015 .0048$

[28] Yusof A, Engelhardt A, Karlsson A et al. Evaluation of a new Etest vancomycinteicoplanin strip for detection of glycopeptide-intermediate Staphylococcus aureus (GISA), in particular, heterogeneous GISA. J Clin Microbiol. 2008; 46:3042-3047. DOI: 10.1128/JCM.00265-08

[29] van Hal SJ, Wehrhahn MC, Barbagiannakos T et al. Performance of various testing methodologies for detection of heteroresistant vancomycin-intermediate Staphylococcus aureus in bloodstream isolates. J Clin Microbiol. 2011; 49:1489-1494. DOI: 10.1128/ JCM.02302-10

[30] Maor Y, Rahav G, Belausov N et al. Prevalence and characteristics of heteroresistant vancomycin-intermediate Staphylococcus aureus bacteremia in a tertiary care center. J Clin Microbiol. 2007; 45:1511-1514. DOI: 10.1128/JCM.01262-06

[31] Etest ${ }^{\circledast}$ Application Guide [Internet]. Available from: http://www.biomerieux-usa.com/ sites/subsidiary_us/files/supplementary_inserts_-_16273_-_b_-_en_-_eag_-_etest_application_guide-3.pdf

[32] Leonard SN, Rossi KL, Newton KL et al. Evaluation of the Etest GRD for the detection of Staphylococcus aureus with reduced susceptibility to glycopeptides. J Antimicrob Chemother. 2009; 63:489-492. DOI: 10.1093/jac/dkn520

[33] Walsh TR, Bolmström A, Qwärnström A et al. Evaluation of current methods for detection of staphylococci with reduced susceptibility to glycopeptides. J Clin Microbiol. 2001; 39:2439-2444. DOI: 10.1128/JCM.39.7.2439-2444.2001

[34] Dhand A, Sakoulas G. Reduced vancomycin susceptibility among clinical Staphylococcus aureus isolates ('the MIC Creep'): implications for therapy. F1000 Med Rep. 2012; 4:4. DOI: $10.3410 / \mathrm{M} 4-4$

[35] Gomes DM, Ward KE, LaPlante KL. Clinical implications of vancomycin heteroresistant and intermediately susceptible Staphylococcus aureus. Pharmacotherapy. 2015; 35:424432. DOI: $10.1002 /$ phar. 1577

[36] van Hal SJ, Paterson DL. Systematic review and meta-analysis of the significance of heterogeneous vancomycin-intermediate Staphylococcus aureus isolates. Antimicrob Agents Chemother. 2011; 55:405-510. DOI: 10.1128/AAC.01133-10

[37] Pillai SK, Wennersten C, Venkataraman L et al. Development of reduced vancomycin susceptibility in methicillin-susceptible Staphylococcus aureus. Clin Infect Dis. 2009; 49:1169-1174. DOI: 10.1086/605636

[38] Rybak MJ, Leonard SN, Rossi KL et al. Characterization of vancomycin-heteroresistant Staphylococcus aureus from the metropolitan area of Detroit, Michigan, over a 22-year period (1986 to 2007). J Clin Microbiol. 2008; 46:2950-2954. DOI: 10.1128/JCM.00582-08 
[39] Di Gregorio S, Perazzi B, Ordoñez AM et al. Clinical, microbiological, and genetic characteristics of heteroresistant vancomycin-intermediate Staphylococcus aureus bacteremia in a teaching hospital. Microb Drug Resist. 2015; 21:25-34. DOI: 10.1089/ mdr.2014.0190

[40] Majcherczyk PA, Barblan JL, Moreillon P et al. Development of glycopeptide-intermediate resistance by Staphylococcus aureus leads to attenuated infectivity in a rat model of endocarditis. Microb Pathog. 2008; 45:408-414. DOI: 10.1016/j.micpath.2008.09.003

[41] Peleg AY, Monga D, Pillai S et al. Reduced susceptibility to vancomycin influences pathogenicity in Staphylococcus aureus infection. J Infect Dis. 2009; 199:532-536. DOI: $10.1086 / 596511$

[42] Claeys KC, Lagnf AM, Hallesy JA et al. Pneumonia caused by methicillin-resistant Staphylococcus aureus: does vancomycin heteroresistance matter? Antimicrob Agents Chemother. 2016; 60:1708-1716. DOI: 10.1128/AAC.02388-15

[43] $\mathrm{Hu} \mathrm{HC}$, Kao KC, Chiu LC et al. Clinical outcomes and molecular typing of heterogenous vancomycin-intermediate Staphylococcus aureus bacteremia in patients in intensive care units. BMC Infect Dis. 2015; 15:444. DOI: 10.1186/s12879-015-1215-2

[44] Wootton M, MacGowan AP, Walsh TR. Comparative bactericidal activities of daptomycin and vancomycin against glycopeptide-intermediate Staphylococcus aureus (GISA) and heterogeneous GISA isolates. Antimicrob Agents Chemother. 2006; 50:4195-4197. DOI: 10.1128/AAC.00678-06

[45] Leonard SN, Rybak MJ. Evaluation of vancomycin and daptomycin against methicillin-resistant Staphylococcus aureus and heterogeneously vancomycin-intermediate $S$. aureus in an in vitro pharmacokinetic/pharmacodynamic model with simulated endocardial vegetations. J Antimicrob Chemother. 2009; 63:155-160. DOI: 10.1093/jac/ dkn439

[46] Kelley PG, Gao W, Ward PB et al. Daptomycin non-susceptibility in vancomycinintermediate Staphylococcus aureus (VISA) and heterogeneous-VISA (hVISA): implications for therapy after vancomycin treatment failure. J Antimicrob Chemother. 2011; 66:1057-1060. DOI: 10.1093/jac/dkr066.

[47] Sievert DM, Rudrik JT, Patel JB et al. Vancomycin-resistant Staphylococcus aureus in the United States, 2002-2006. Clin Infect Dis. 2008; 46:668-674. DOI: 10.1086/527392

[48] Howden BP, Ward PB, Charles PG et al. Treatment outcomes for serious infections caused by methicillin-resistant Staphylococcus aureus with reduced vancomycin susceptibility. Clin Infect Dis. 2004; 38:521-528. DOI: 10.1086/381202

[49] Bishop E, Melvani S, Howden BP et al. Good clinical outcomes but high rates of adverse reactions during linezolid therapy for serious infections: a proposed protocol for monitoring therapy in complex patients. Antimicrob Agents Chemother. 2006; 50:15991602. DOI: 10.1128/AAC.50.4.1599-1602.2006 
[50] Sun W, Chen H, Liu Y et al. Prevalence and characterization of heterogeneous vancomycin-intermediate Staphylococcus aureus isolates from 14 cities in China. Antimicrob Agents Chemother. 2009; 53:3642-3649. DOI: 10.1128/AAC.00206-09

[51] Saravolatz LD, Pawlak J, Johnson LB. In vitro susceptibilities and molecular analysis of vancomycin-intermediate and vancomycin-resistant Staphylococcus aureus isolates. Clin Infect Dis. 2012; 55:582-586. DOI: 10.1093/cid/cis492 